\title{
Twisting Fluorescence through Extrinsic Chiral Antennas
}

\author{
Chen Yan, ${ }^{\circledR}$ Xiaolong Wang, T. V. Raziman, ${ }^{\circledR}$ and Olivier J. F. Martin*(1) \\ Nanophotonics and Metrology Laboratory, Swiss Federal Institute of Technology (EPFL), CH-1015 Lausanne, Switzerland
}

\section{Supporting Information}

ABSTRACT: Plasmonic antennas and planar structures have been undergoing intensive developments in order to control the scattering and absorption of light. One specific class, extrinsic chiral surfaces, that does not possess 2 -fold rotational symmetry exhibits strong asymmetric transmission for different circular polarizations under obliquely incident illumination. In this work, we show that the design of those surfaces can be optimized with complex multipolar resonances in order to twist the fluorescence emission from nearby molecules. While this emission is usually dipolar and linearly polarized, the interaction with these resonances twists it into a multipolar
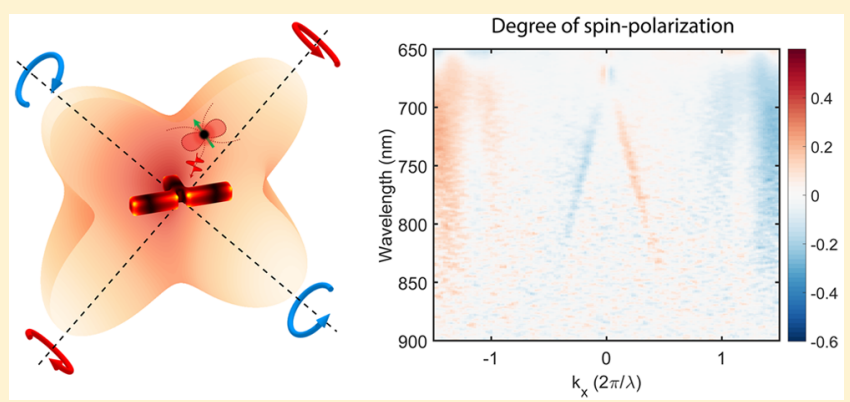
radiation pattern with opposite helicity in different directions. The proposed structure maximizes this effect and provides control over the polarization of light. Splitting of left- and right-handed circularly polarized light is experimentally obtained in the backward direction. These results highlight the intricate interplay between the near-field absorption and the far-field scattering of a plasmonic nanostructure and are further used for modifying the emission of incoherent quantum sources. Our finding can potentially lead to the development of polarization- and angle-resolved ultracompact optical devices.

KEYWORDS: Plasmonic nanoantennas, multipolar resonances, extrinsic chirality, $k$-space polarimetry, fluorescence emission

$\mathrm{P}$ lasmonic nanoantennas are a very effective platform for mastering light-matter interaction, offering an exceptional degree of freedom in manipulating the polarization state and phase of light. ${ }^{1-10}$ Complex polarization-related phenomena have especially been observed in chiral metasurfaces built from nanoantennas; these phenomena include spin Hall effects, ${ }^{11-15}$ spin-controlled wavefront shaping, ${ }^{16,17}$ polarization-dependent excitation of waveguide mode/SPPs, ${ }^{18,19}$ polarization conversion, ${ }^{20-23}$ asymmetric transmission, ${ }^{24-27}$ and extraordinary circular dichroism (CD) ${ }^{28,29}$ In addition, achiral structures in conjunction with strong birefringence can also produce polarization-dependent transmission thanks to their supported high order resonances. ${ }^{30,31}$ In that case, the absorption is different between left- and right-handed circular polarization (LCP, RCP) at oblique incidence, generating strong circular dichroism known as extrinsic chirality. ${ }^{31-33}$

Furthermore, an antenna can affect the scattering from nearby quantum emitters. ${ }^{10,34,35}$ In addition to enhancing the spontaneous emission rate through an increase in the local density of states, ${ }^{36-39}$ the far-field scattering pattern is modified as well, ${ }^{40-42}$ and plasmonic antennas have been employed to achieve intense and highly directional emission from fluorescent molecules and quantum dots. ${ }^{43-47}$ Planar chiral structures can alter the far-field polarization from fluorescent emitters and give rise to asymmetric intensities of LCP and RCP light emitted from the sample. ${ }^{48,49}$ Multipolar effects inherent to complex optical antennas have recently attracted special attention in nonlinear nanophotonics as well as directional fluorescence emission. ${ }^{44,50}$ However, the confinement in angular distribution of fluorescence is often restricted to the broad radiation of the antenna depending on the highest order multipolar component. The combination of narrow emission channel and circularly polarized emission based on achiral structures is highly desirable. This can be achieved by utilizing an extrinsic chiral metasurface incorporating a planar waveguide as will be discussed in this work.

We report a plasmonic planar surface made out of T-shaped antennas with maximized extrinsic chiral response at oblique incidence and experimentally demonstrate polarization-modified emission from nearby incoherent fluorescent sources. We directly observe the efficient splitting of circularly polarized light, similar to the photonic spin Hall effect. ${ }^{11}$ Different from other diffraction-based phase varying surfaces, the effect here results from the interplay of multipolar modes from individual antennas and is driven by near-field interactions. Specifically, we use vector spherical harmonics to extract the modes of the T-shaped antenna and show that it simultaneously supports an electric dipole mode and an electric quadrupole mode. By carefully designing the shape of the antenna, pure circularly polarized scattering can be achieved in the out-of-plane direction. We experimentally measure the degree of circular polarizability (DCP) using a Fourier imaging technique ${ }^{51}$ and observe circularly polarized light with opposite handedness at different angles in the backward direction. We further show that plane-wave excitation at these particular angles leads to pronounced circular dichroism and asymmetric extinction.

Received: November 24, 2016

Revised: March 16, 2017

Published: March 17, 2017 


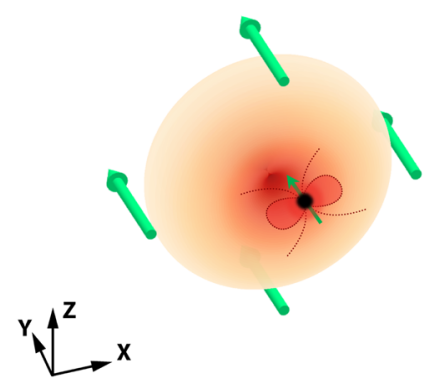

C

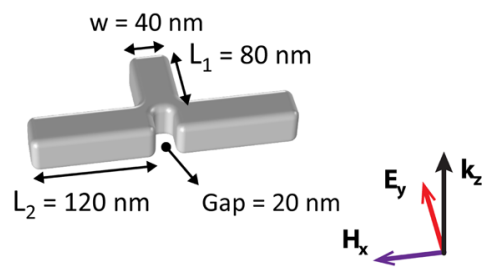

e

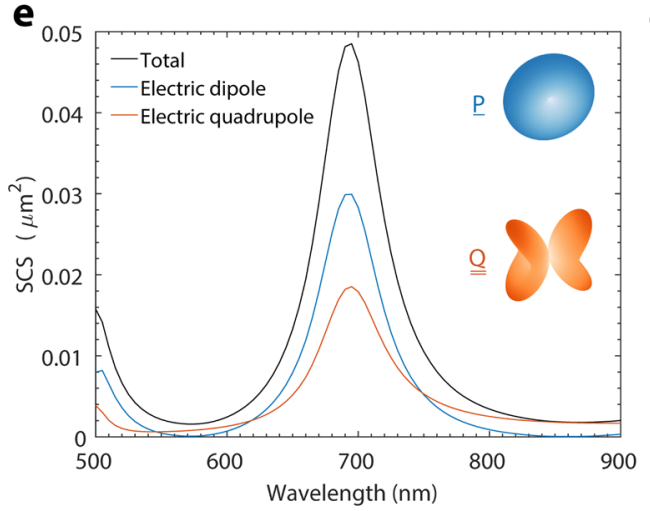

b

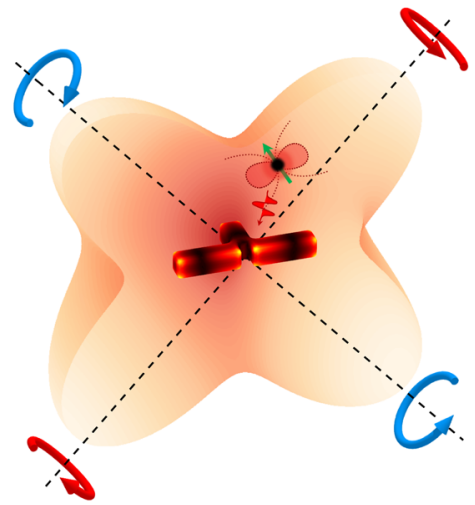

d

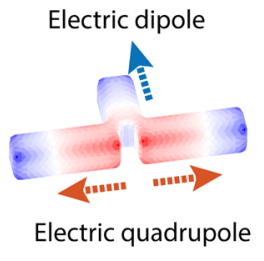

f

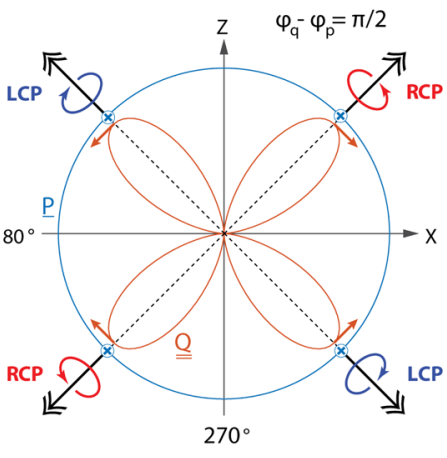

Figure 1. (a) Radiation pattern for a dipole emitter: the far-field emission is linearly polarized in all directions (green arrows). (b) Enhanced radiation pattern for a dipole emitter placed in the hotspot of a chiral antenna excited by $y$-polarized light: the light is directed into four narrow lobes with opposite circular polarizations (red circles for RCP; blue circles for LCP). (c) Schematic of the proposed T-shaped nanoantenna illuminated by a $y$-polarized plane wave propagating in the $z$-direction. (d) Computed charge distribution at the resonance wavelength $\lambda=690 \mathrm{~nm}$. (e) Simulated scattering spectrum for a single antenna (black curve), and the contributions from the electric dipole (blue curve), and the electric quadrupole (red curve) computed using a vector spherical harmonic (VSH) decomposition. (f) Radiation pattern in the $x z$-plane from the electric dipole and quadrupole modes (in blue and red, respectively).

Lastly, we establish a link between the near-field absorption and far-field emission and demonstrate that metasurfaces with strong extrinsic chirality can be used for controlling the circularly polarized emission from achiral fluorophores in the vicinity of the plasmonic antennas. Our results have practical implications for designing the optical activity in planar materials and can be used to selectively enhance the radiation of circularly polarized light from single emitters.

Design Principle. It is known that a dipole emitter radiates light in a donut-shaped pattern as shown in Figure 1a. The radiation in the azimuthal plane is isotropic and linearly polarized parallel to the dipole moment. Near-field coupling with antenna allows efficient extraction of light into the far-field. Along with the increased intensity, the radiation pattern is modified and the polarization of the antenna-altered far-field does not necessarily remain linear. It is thus possible to engineer both the angular intensity and polarization of the emitted light to achieve novel functionalities. In this article, we carefully designed a T-shaped antenna that twists the power flow into perfect circular polarization and directs it into four narrow lobes, as illustrated in Figure $1 \mathrm{~b}$. It consists of a nanorod attached perpendicular to a gap antenna (Figure 1c): the gap antenna in the $x$-direction has two arms of $120 \mathrm{~nm}$ in length and $40 \mathrm{~nm}$ in width and thickness with a gap of $20 \mathrm{~nm}$, and an $80 \mathrm{~nm}$ long nanorod in the $y$-direction is attached to the gap with an overlap of $10 \mathrm{~nm}$. Briefly, this perpendicular nanorod alone supports a dipole mode when illuminated with $y$-polarized light. It acts as a seed for exciting the antibonding mode of the gap antenna that is symmetry-forbidden under normal plane wave illumination. The combination of the electric dipole and quadrupole allows the generation of circularly polarized light in directions satisfying the required phase and amplitude matching conditions. We simulate the optical response using the surface integral equation $(\mathrm{SIE})^{52}$ method (details in the Methods section). The scattering spectrum is plotted as a black curve in Figure 1e, where a resonance peak is visible at $\lambda=690 \mathrm{~nm}$. 

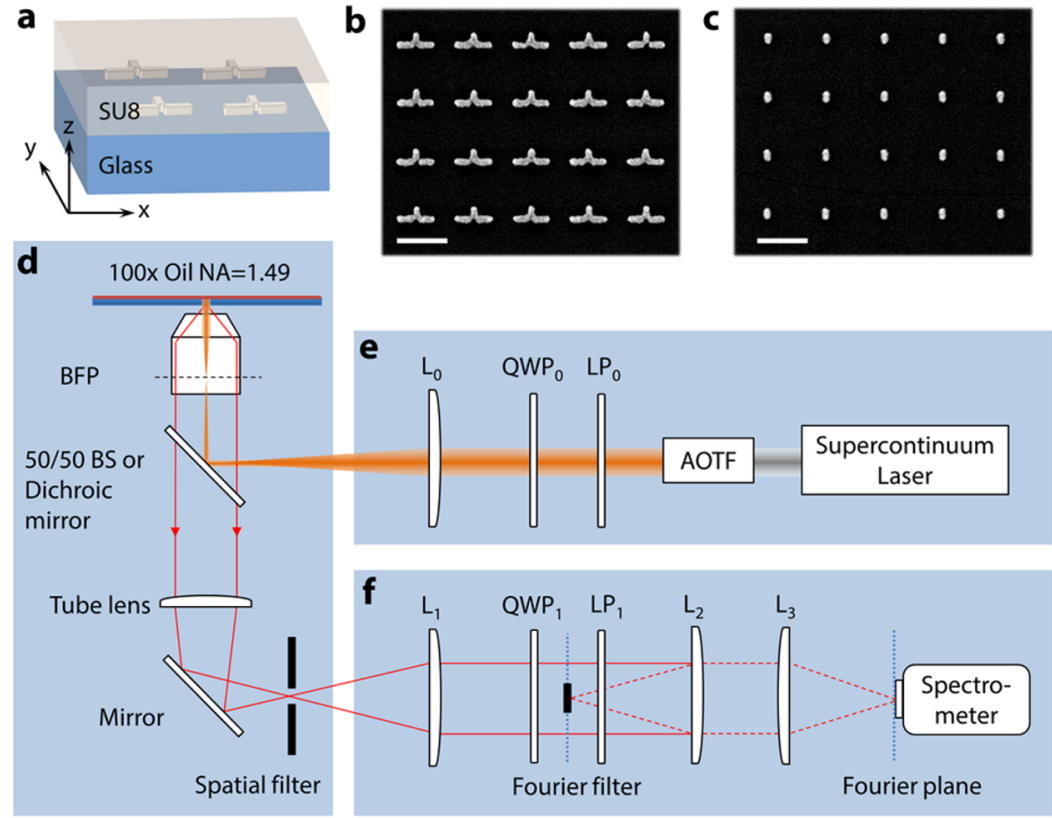

Figure 2. (a) Schematic of the sample, a $400 \mathrm{~nm}$ of SU8 cladding is spin-coated on silver nanostructures supported by a glass substrate. SEM images of the arrays of (b) T-shaped antennas and (c) nanorods along $y$-direction. Scale bars correspond to $400 \mathrm{~nm}$. (d-f) Schematic of the setup. The polarization of the illumination path (e) is controlled by a linear polarizer $\left(\mathrm{LP}_{0}\right)$ and a quarter-wave plate $\left(\mathrm{QWP}_{0}\right)$. The laser is focused into an inverted microscope $(\mathrm{d})$ with a $100 \times$ objective. In the detection path $(\mathrm{f})$, polarization is analyzed by another quarter-wave plate $\left(\mathrm{QWP}_{1}\right)$ and a linear polarizer $\left(\mathrm{LP}_{1}\right)$. The dark-field image is formed by filtering the Fourier plane and then the spectral information is resolved by a spectrometer.

To understand unambiguously the radiation properties of this resonance, we perform the field multipole expansion using the orthogonality of the vector spherical harmonics (VSH), ${ }^{53}$ see Supporting Information for details. In Figure 1e, scattering cross-section is decomposed into different multipolar modes with the blue line representing the electric dipole contribution $\underline{\mathbf{P}}$ and the orange line representing the electric quadrupole contribution $\underline{\mathbf{Q}}$. Note that at the wavelength $\lambda=690 \mathrm{~nm}$, these two orthogonal modes are spectrally superimposed with the $Q_{x x}$ component dominating the quadrupole moment and the $P_{y}$ component dominating the dipole moment. The corresponding emission patterns for these two modes are presented as insets in Figure 1e (blue for the dipole emission and orange for the quadrupolar emission). The charge distribution at this wavelength (in Figure 1d) further confirms an optical response with a dipolar oscillation on the perpendicular nanorod and an antibonding distribution in the two long arms exhibiting a quadrupolar characteristic.

Let us consider in Figure $1 \mathrm{f}$ the radiation intensity in the $x z$ plane for the electric dipole and the electric quadrupole, computed at this resonance wavelength. The electric dipole $P_{y}$ produces isotropic emission in $x z$-plane (blue curve) with electric field along the $y$-axis (cross marker). On the other hand, the electric quadrupole with dominant component $Q_{x x}$ results in a four-lobed pattern in the $x z$-plane with electric field along the polar direction (orange curve and arrow). We specifically tune the lengths of the antenna $L_{1}$ and $L_{2}$ such that the amplitude of electric fields from the dipole and the quadrupole at $45^{\circ}, 135^{\circ}, 225^{\circ}$ and $315^{\circ}$ match each other. Even though the amplitudes are identical at these four particular angles, the sum of the scattering field over all solid angles for the quadrupole mode is still smaller than that of the dipole mode, hence the lower scattering cross-section as presented in Figure 1e. In addition, there is a $\pi / 2$ phase difference between electric farfield from these two modes and, consequently, circularly polarized radiation is obtained. The opposite handedness with respect to the $x$ - and $z$-axis is due to the geometric symmetry of the antenna in these directions and satisfies the conservation of spin angular momentum. Overall, although the incident light is purely linearly polarized, conversion to circular polarization is achieved thanks to the coupling to the quadrupole mode. The exact value of DCP as a function of three-dimensional detection angle is presented in Figure S1 of Supporting Information.

Measurements and Results. Experimentally, we study silver $\mathrm{T}$-shaped antennas fabricated on a glass substrate and embedded in a $400 \mathrm{~nm}$ thick SU8 layer, as illustrated in Figure 2a (for the sample preparation, see Method; the SU8 layer will be required later for fluorescence measurements). We consider successively individual antennas and arrays of antennas; Figure $2 \mathrm{~b}$ shows, for example, the SEM images of a fabricated array of antennas with periodicity $435 \mathrm{~nm}$. A reference array, which consists of only nanorods in the $y$-direction, is also fabricated for comparison (Figure 2c). The measurements are performed on a homemade $k$-space polarimetry setup as illustrated in Figure $2 \mathrm{~d}-\mathrm{f}$. In the excitation path (Figure 2e), a supercontinuum together with an acousto-optical tunable filter (AOTF) is used for either broadband or narrowband illumination. A linear polarizer $\left(\mathrm{LP}_{0}\right)$ and a quarter-wave plate $\left(\mathrm{QWP}_{0}\right)$ are used for controlling the incidence polarization. Then, the light is focused on the center of the back focal plane (BFP) of a $100 \times$ oil immersion objective with NA = 1.49 and results in an excitation beam normal to the sample (Figure 2d). Moving focus position in the BFP allows changing the incident angle from $-78^{\circ}$ to $78^{\circ}$. In the detection path (Figure 2f), a circular shape filter is placed in the Fourier plane to block the excitation light. Another set of a quarter-wave plate $\left(\mathrm{QWP}_{1}\right)$ and a linear polarizer $\left(\mathrm{LP}_{1}\right)$ is employed for polarimetry analysis. Finally, we measure the angular distribution and polarization state by imaging the Fourier plane on the CCD of the spectrometer. Such a configuration 

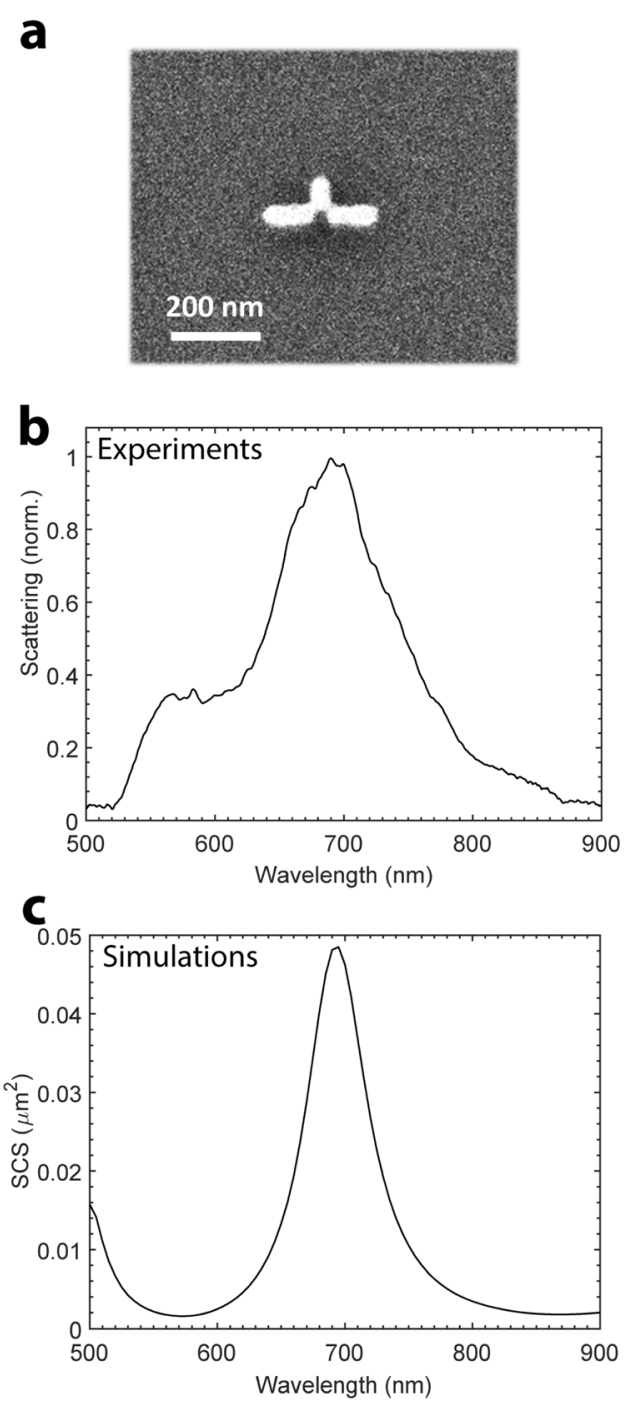
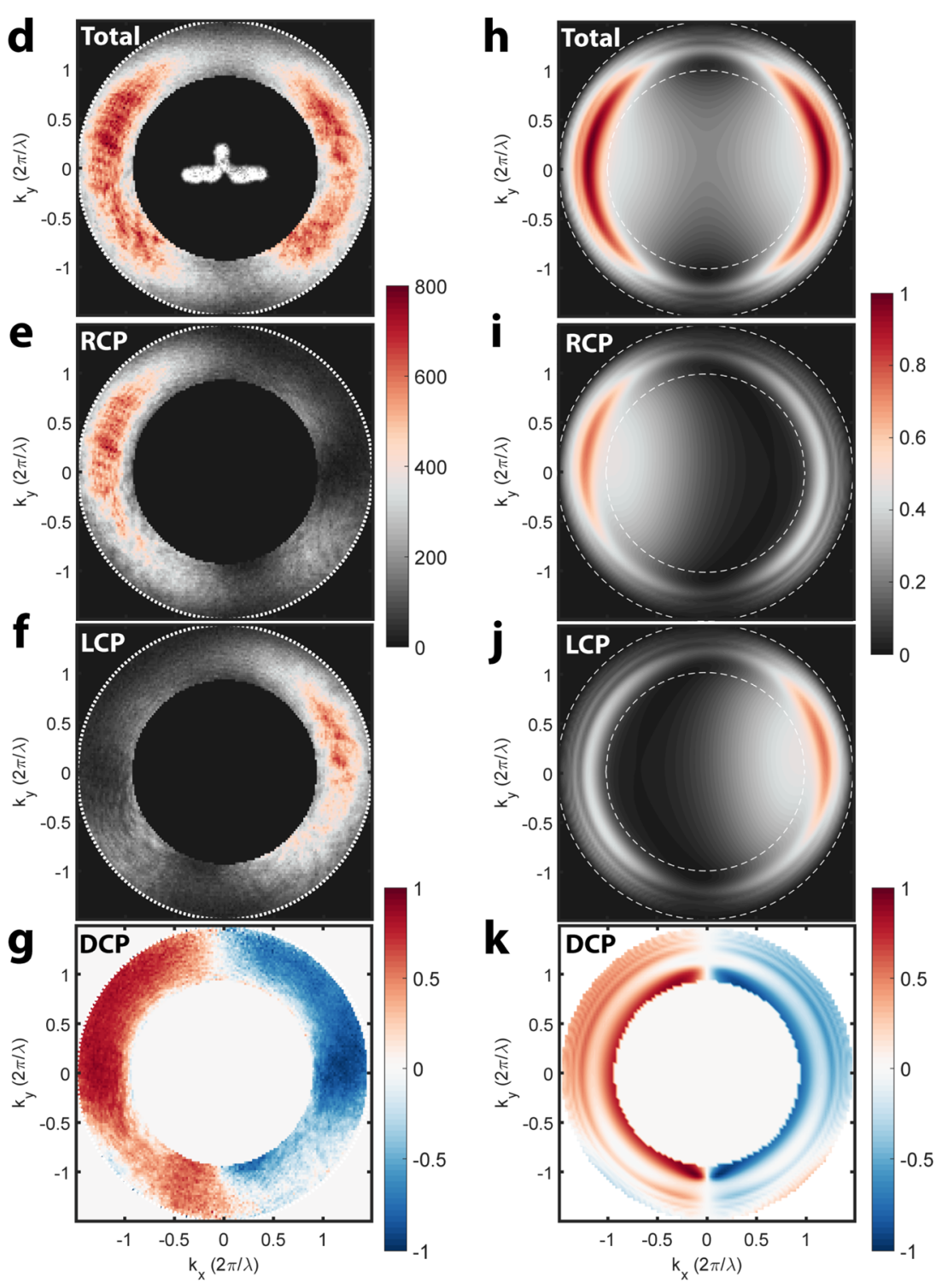

Figure 3. (a) SEM image of an individual T-shaped antenna. (b) Measured and (c) simulated scattering spectra from a single antenna. (d-g) Measured radiation pattern in the Fourier plane for the single T-shaped antenna: (d) total intensity, (e) intensity of right-handed circular polarization (RCP), (f) intensity of left-handed circular polarization (LCP), and (g) degree of circular polarization (DCP). (h-k) Corresponding quantities calculated from the simulation.

allows us to measure the backward scattering pattern for the $z<$ 0 half-space.

We first perform dark-field scattering spectral measurements on a single $\mathrm{T}$-shaped antenna, Figure $3 \mathrm{~b}$, where a scattering peak is observed at $690 \mathrm{~nm}$ in good agreement with the simulation, Figure 3c. As we are interested in the angular and polarization properties of the resonance, we excite the structure with monochromatic light with $\lambda=690 \mathrm{~nm}$ and measure the Fourier plane image under different angular combinations of $\mathrm{QWP}_{1}$ and $\mathrm{LP}_{1}$. Here, we define the degree of circular polarization (DCP) using the Stokes parameters as DCP $=S_{3}$ / $S_{0}$, where DCP $=1$ and -1 correspond to right-handed circular polarization (RCP) and left-handed circular polarization $(\mathrm{LCP})^{54}$ (details of Stokes parameters measurements in the Supporting Information). The results are summarized in Figure $3 \mathrm{~d}-\mathrm{g}$, where the center black area is due to the dark-field filter in the Fourier plane. First of all, the total scattering intensity $S_{0}$ shows a typical dipolar-like pattern with $P_{y}$ dominant. The electric field is minimal at a large angle in $y$-direction and mirror-symmetric with respect to the $y$-axis. Two maximal scattering lobes appear between wavevectors $\left|k_{x}\right|=1$ and 1.5 due to the effect of the air/cladding interface. ${ }^{55}$ Figure $3 \mathrm{e}, \mathrm{f}$ shows the measured intensity for RCP and LCP light. These reveal that each scattering lobe exhibits its own handedness of circular polarization and their DCP show opposite signs (Figure $3 \mathrm{~g}$ ). Such a scattering property with strong intensity in the $+x$ and $-x$ side of the backward direction and opposite handedness is consistent with the analysis in Figure if. The calculated Fourier plane images including the substrate effect are plotted in Figure $3 \mathrm{~h}-\mathrm{k}$ and match very well with the measurements. The outer rim corresponds to the NA of the objective and the inner dashed ring corresponds to the critical angle for total internal reflection.

Given these polarization properties, the T-shaped antenna we designed here is an ideal unit-cell to construct extrinsic chiral periodic surfaces. The periodic array we study now has periodicity $p=435 \mathrm{~nm}$, as shown in the SEM image in Figure $2 \mathrm{~b}$. We characterize the extinction of the surface by taking the 

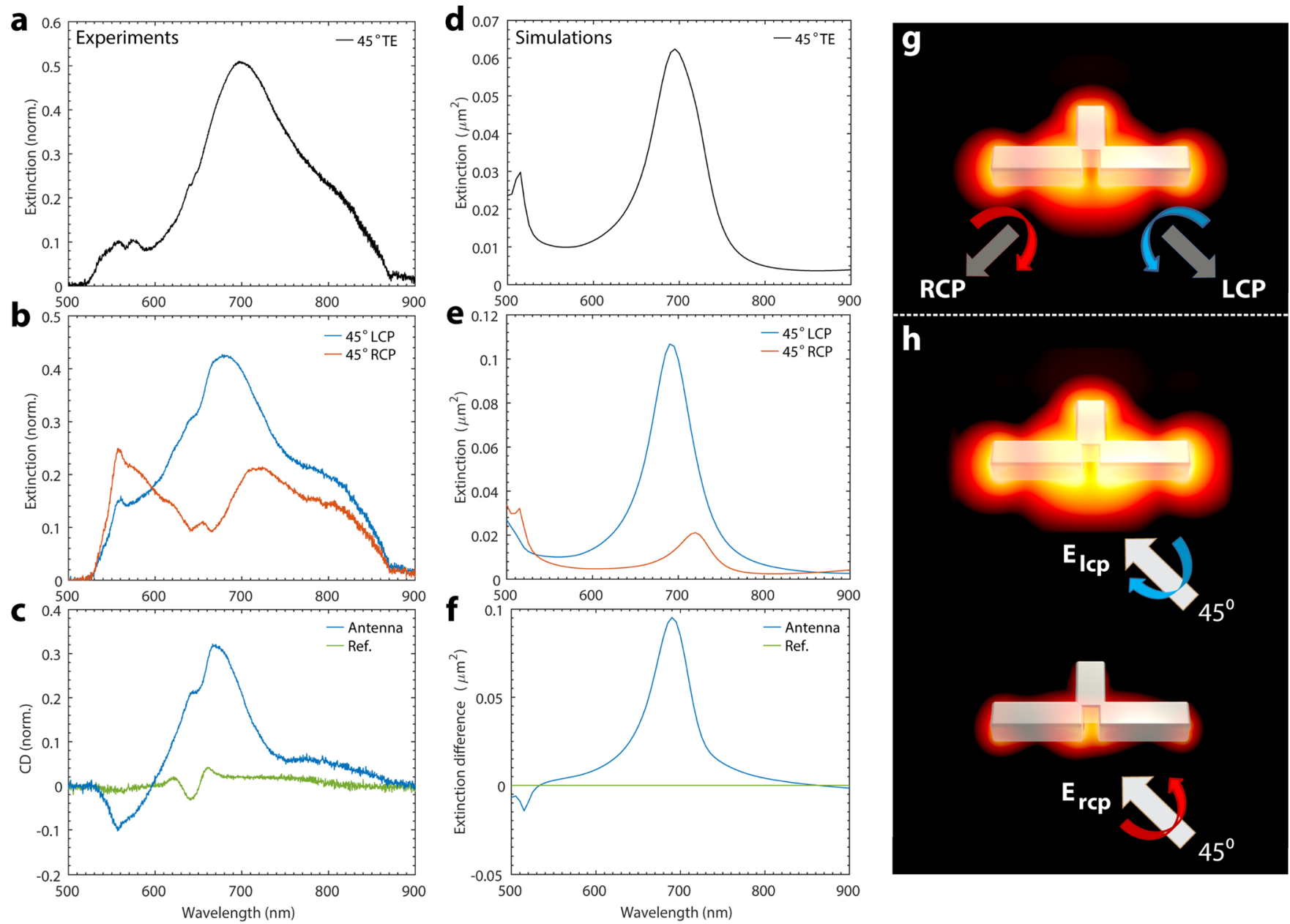

Figure 4. (a,b) Experimental extinction spectra of an array of T-shaped antenna for an incidence at $45^{\circ}$ with (a) TE polarization and (b) LCP and RCP polarization in blue and red curves, respectively. (c) CD spectra from an array of T-shaped antennas (blue curve) and a reference nanorods array (green curve). ( $\mathrm{d}-\mathrm{f})$ Simulated extinction cross-section of a single antenna for the corresponding illumination condition. (g,h) Computed near-field intensity in the same log 10 scale (from red to yellow) for (g) normal incidence and (h) incidence at $45^{\circ}$ with LCP and RCP polarizations.

difference between reflection from the antenna array and reflection without nanostructures at oblique incidence. (Thanks to the experimental configuration, for oblique incidence at $45^{\circ}$ in $x z$-plane, light is totally internally reflected.) For transverse electric (TE) polarization (incident electric field normal to the plane of incidence), the extinction is shown in Figure 4a. Strong absorption is observed at a wavelength around $700 \mathrm{~nm}$, which coincides with the scattering peak of a single antenna. The resonance is slightly broader due to the inhomogeneous broadening caused by the array structure. Interestingly, the extinction spectra at this incident angle are very different for LCP and RCP light, as shown in Figure 4b. The LCP extinction spectrum remains similar to the one with TE polarization. However, in the case of RCP (red curve in Figure 4b) an extinction dip appears at $690 \mathrm{~nm}$ indicating that such illumination condition cannot efficiently excite the multipolar mode of the T-shaped antenna. Under oblique incidence, the structure does not hold mirror symmetry and thus yields a strong circular dichroism as plotted in Figure 4c. The blue line corresponds to the extinction difference from the $\mathrm{T}$-shaped antenna planar array and the green curve corresponds to that of the reference nanorod array. A contrast as high as $30 \%$ is obtained in this configuration for the T-shaped antenna array. The small variation at $640 \mathrm{~nm}$ is due to coupling to the diffracted order of the Wood-Rayleigh anomaly, which enhances the $\mathrm{CD}$ signal lifted by the symmetry breaking in the $z$-direction of the glass interface. ${ }^{56,57}$ All the extinction measurements match very well with the simulation in Figure $4 \mathrm{~d}-\mathrm{f}$ for the cases of TE polarization, circular polarization, and extinction difference.

Similar behavior, named extrinsic chirality, has been previously reported in other planar metamaterials. ${ }^{33}$ This effect is especially strong in the case of the T-shaped antenna. The $\mathrm{CD}$ response that we observed can be related to the polarization state of the scattering from the antenna. From the above study, we know that $y$-polarized light excites a quadrupolar mode which generates LCP at $45^{\circ}$; while $x$ polarized light excites a bonding mode which generates radiation with much lower DCP at this angle (see Supporting Information for details). Using the reciprocity theorem, this implies that left- and right- circularly polarized light incident at $45^{\circ}$ excite the bonding mode almost equally, but excite the quadrupolar mode unequally. This difference results in the CD response. The near-field enhancement further provides a qualitative understanding of the strength of the resonance under different excitation conditions. Figure $4 \mathrm{~g}$ shows the calculated near-field in logarithmic scale under normal $y$ polarized incidence, which radiates LCP light at $45^{\circ}$ in the 

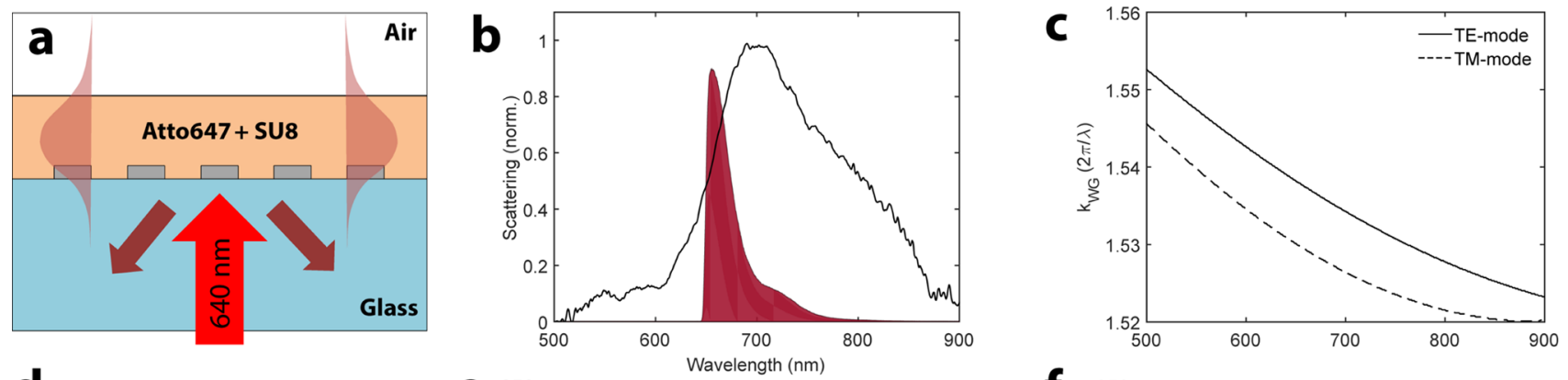

d
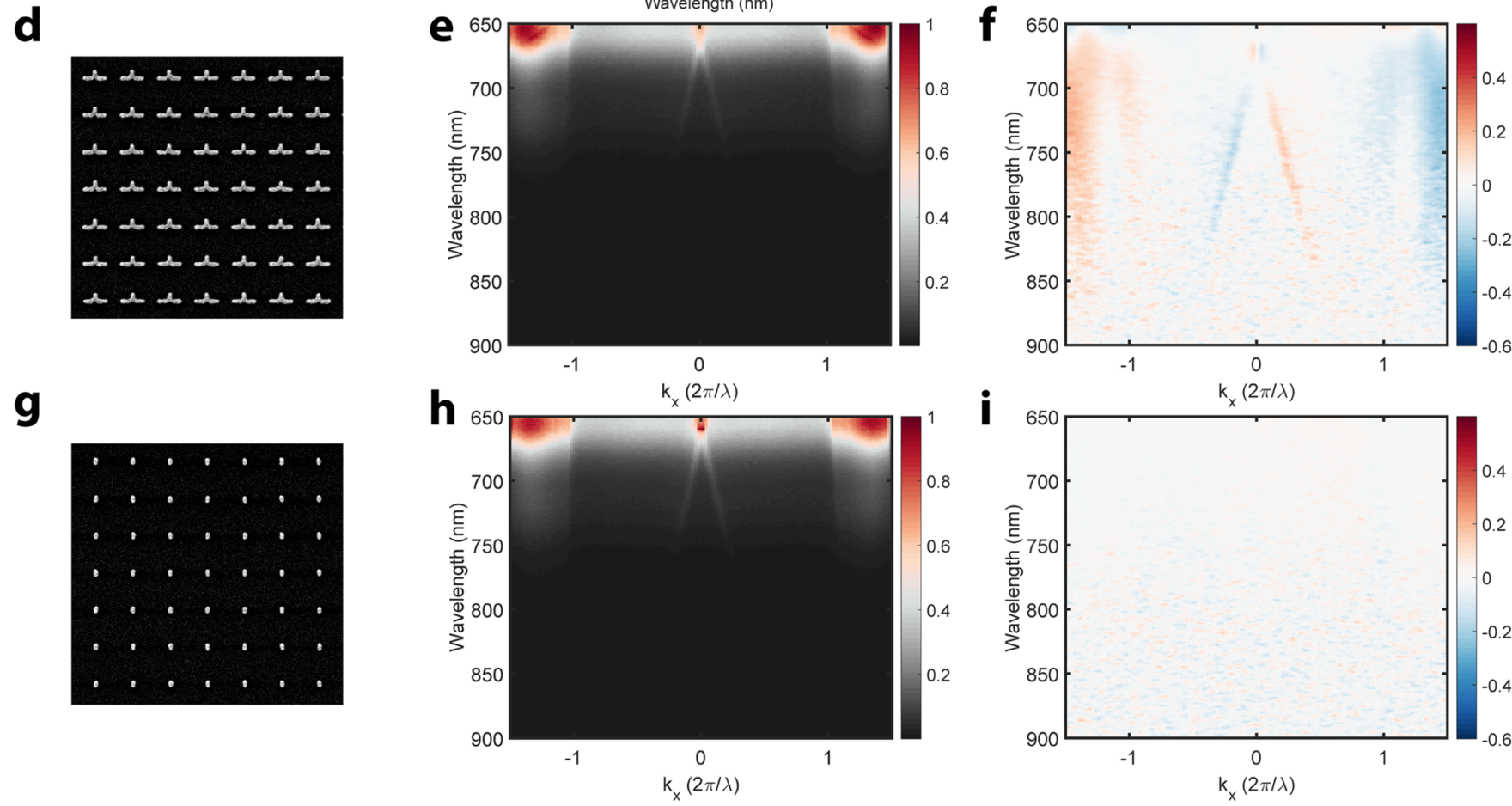

Figure 5. (a) Schematic of the sample spin-coated with fluorescent molecules ATTO647N embedded in the $400 \mathrm{~nm}$ thick SU8 cladding. (b) The emission spectrum of the molecule (dark red shaded area) overlaps with the measured dark-field scattering spectrum from the T-shaped array (black curve). (c) Dispersion relation for the guided modes supported by the SU8 slab: TE (black solid line) and TM (black dashed line). (d) SEM image of the T-shaped antenna array measured in the experiment. (e,f) Measured dispersion relation for (e) the fluorescence intensity and (f) the DCP of the emitted light. (g) SEM image of the reference array. (h,i) Measured dispersion relation for $(\mathrm{h})$ the fluorescence intensity and (i) the DCP of fluorescence emission.

backward direction. As a consequence, when illuminating at $45^{\circ}$ (Figure 4h), only LCP light produces strong near-field intensity (upper graph), while near-field is extremely weak for the RCP (lower graph).

Since this strong absorption and circularly polarized scattering occur at the individual antenna level, this planar Tshaped antenna array is extremely efficient in extracting the fluorescence and controlling the circular-polarized emission concurrently. To demonstrate the polarization-modified emission from the dye distributed in the vicinity of the antenna array, we prepared the sample with a cladding SU8 mixed with fluorescent dye ATTO647N as illustrated in Figure 5a. The molecule chosen has absorption at $640 \mathrm{~nm}$ and emission above $650 \mathrm{~nm}$, as indicated by the measured emission band in Figure $5 b$ (shaded red area). The fluorescence emission overlaps with the resonance of the T-shaped array (black curve in Figure $5 b$ ). The thickness of the cladding is $400 \mathrm{~nm}$ and SU8 has a refractive index of 1.60. This high index cladding supports wellconfined guided modes, which helps collect the emission from incoherent emitters. The dispersion relations for the TE and TM waveguide modes calculated by transfer matrix method
$(\mathrm{TMM})^{54}$ are plotted in Figure $5 \mathrm{c}$; these are the only modes that exist in this wavelength range. In the experimental setup, we replaced the $50 / 50$ beam splitter and the dark-field filter by a short-pass filter, a dichroic mirror, and a long-pass filter in order to perform fluorescence measurements.

In Figure 5e,h, fluorescence intensity is measured as a function of wavelength and in-plane wavevector $k_{x}$ in the $x$ direction from two arrays: one with T-shaped antennas (Figure 5d) and the other with reference nanorods with 2 -fold symmetry (Figure $5 \mathrm{~g}$ ). The fluorescence twisted by the sample is mainly distributed into two parts: two narrow angular emission bands observed at low $k_{x}$, corresponding to the fluorescence excitation of TE and TM waveguide modes, which are then coupled to the glass substrate due to the diffraction from the periodic antenna array (see Supporting Information for details); broad angular emission at $\left|k_{x}\right|$ larger than the total internal reflection angle comes from the molecules in the vicinity of the antenna with their emission modified by the plasmonic resonance. Although the fluorescence intensity distributes identically for both arrays, the difference can be distinguished by analyzing the DCP of the emitted light, as 
measured in Figure 5f,i. For the T-shaped antenna array (Figure $5 f)$, we observe RCP light in the $-x$ direction and LCP light in the $+x$ direction for $\left|k_{x}\right|>1$. This behavior is similar to the radiation of a single antenna, indicating that the emission of molecules is strongly modified by coupling to the plasmonic resonances. Furthermore, the diffraction of long-range guided modes propagating in $+x$ and $-x$ directions generates two highly directed emission bands close to the normal $\left(k_{x} \approx 0\right)$ direction, exhibiting opposite handedness (details of fitting of diffraction orders in Supporting Information). Here, the maximal DCP is reduced to approximately 0.5 due to high background intensity from molecules present in the SU8 slab, Figure 5f, while it reached 1 for the light scattered by the structure alone, Figure 3g. On the contrary, such spin-polarized response is totally absent in the fluorescence from the reference nanorod array (Figure 5i).

Conclusions. In summary, we have introduced a new Tshaped plasmonic structure that supports both electric dipolar and quadrupolar modes. The interplay between these orthogonal modes can generate remarkable circularly polarized radiation in particular directions. Linear excitation is converted into RCP and LCP which is then redirected to $\pm 45^{\circ}$ in out-ofplane directions. This behavior is impossible with conventional dipole antennas. Using the reciprocity theorem, we find a strong correlation between the extrinsic chirality and the circularly polarized emission. A periodic array of such antennas is proven to exhibit a maximized extrinsic chirality at $45^{\circ}$ enabled by the nontrivial angle-resolved radiation of its building block. By coupling with achiral fluorescent molecules, direct generation and splitting of circularly polarized light from this planar surface is achieved. We experimentally realized opposite handedness in different emission directions, which solely relies on the properties of the T-shaped plasmonic antennas. Our results establish a general basis for controlling the polarization state and emission angle from incoherent quantum emitters using planar plasmonic structures. This control of polarization is key to advanced imaging techniques such as stimulated emission depletion (STED) ${ }^{58}$ or differential interference contrast (DIC) ${ }^{59}$ microscopies; this work could take these techniques to the next level as well as enabling new polarization-dependent light-emitting devices for applications in imaging, optical communication, optical displays, sensing, and spectroscopy.

Methods. Fabrication and Sample Preparation. The silver antennas were fabricated on top of a $150 \mu \mathrm{m}$ thick float glass substrate using a previously introduced method. ${ }^{60}$ Briefly, a double-layer PMMA photoresist coating is exposed with electron beam lithography (Vistec EBPG5000 system) using a $100 \mathrm{keV}$ electron beam. The nanostructures were obtained by thermal evaporation of $1 \mathrm{~nm}$ silver oxide adhesion layer and 39 $\mathrm{nm} \mathrm{Ag}$ followed by a lift-off process. The fabricated arrays cover an area of $40 \times 40 \mu \mathrm{m}^{2}$. To deposit the cladding layer with fluorescent molecules, $0.1186 \mathrm{mM}$ of ATTO647N molecules was dissolved in cyclopentanone and then mixed with SU8 (GM1040) in a 1:1 ratio. Spin-coating the solution on the sample at $6000 \mathrm{rpm}$ for $60 \mathrm{~s}$ resulted in a $\sim 400 \mathrm{~nm}$ thick SU8 layer. The sample was baked at $90{ }^{\circ} \mathrm{C}$ for $2 \mathrm{~min}$ to vaporize the remaining cyclopentanone.

Fourier Imaging Setup. A Fourier imaging setup for spectral-angular measurements was built from a commercial inverted microscope (Olympus IX73). A supercontinuum light source (Fianium FemtoPower 1060) combined with an 8channel acousto-optical tunable filter (Fianium AOTF-V1-N1-
DD) was used for different illumination conditions. The objective has a high numerical aperture with $\mathrm{NA}=1.49$ (Olympus UAPO N 100×/1.49 oil). Achromatic quarter-wave plate is used for the range $450-800 \mathrm{~nm}$ (Thorlabs AQWP05M630). In the case of fluorescence measurement, a high-quality short-pass filter (Thorlabs FESH0650), a dichroic mirror (Chroma ZT640rdc-UF1), and a long-pass filter (Chroma ET655lp) were used to completely remove the excitation light.

Numerical Method. The simulations of nanostructures and array in the homogeneous medium were performed using a custom-developed numerical program based on the SIE technique; ${ }^{52,61}$ the same technique has been used to compute the emission from fluorescent molecules. ${ }^{42}$ The Fourier plane pattern of a single structure on the glass substrate was calculated based on a volume integral equation method incorporating the Green's tensor for stratified media. ${ }^{62}$

\section{ASSOCIATED CONTENT}

\section{Supporting Information}

The Supporting Information is available free of charge on the ACS Publications website at DOI: 10.1021/acs.nanolett.6b04906.

Stokes parameters analysis and fitting of diffraction curves in the fluorescence k-space measurement (PDF)

\section{AUTHOR INFORMATION}

\section{Corresponding Author}

*E-mail: olivier.martin@epfl.ch. ORCID $\odot$

Chen Yan: 0000-0002-3171-0449

T. V. Raziman: 0000-0002-7085-6934

Olivier J. F. Martin: 0000-0002-9574-3119

\section{Author Contributions}

C.Y. conceived the experiment, put together the Fourier imaging setup, and performed all optical measurements; X.W. and C.Y. fabricated the samples; T.V.R. and C.Y. performed the numerical simulations. All authors contributed to the manuscript and gave their approval to the final version.

\section{Funding}

Funding from the European Research Council (ERC-2015AdG-695206 Nanofactory) and from the Swiss National Science Foundation (Project 200021_162453 and 200020_135452) are gratefully acknowledged.

Notes

The authors declare no competing financial interest.

\section{ABBREVIATIONS}

VSH, vector spherical harmonics; LCP, left-handed circular polarization; RCP, right-handed circular polarization; DCP, degree of circular polarization; QWP, quarter wave plate; LP, linear polarizer; BFP, back focal plane; $\mathrm{CD}$, circular dichroism

\section{REFERENCES}

(1) Muhlschlegel, P.; Eisler, H.-J.; Martin, O. J. F.; Hecht, B.; Pohl, D. W. Science 2005, 308, 1607-1608.

(2) Fischer, H.; Martin, O. J. F. Opt. Express 2008, 16, 9144-9154.

(3) Yu, N.; Genevet, P.; Kats, M. A.; Aieta, F.; Tetienne, J.-P.; Capasso, F.; Gaburro, Z. Science 2011, 334, 333-337.

(4) Seok, T. J.; Jamshidi, A.; Kim, M.; Dhuey, S.; Lakhani, A.; Choo, H.; Schuck, P. J.; Cabrini, S.; Schwartzberg, A. M.; Bokor, J.; Yablonovitch, E.; Wu, M. C. Nano Lett. 2011, 11, 2606-2610. 
(5) Abb, M.; Albella, P.; Aizpurua, J.; Muskens, O. L. Nano Lett. 2011, 11, 2457-2463.

(6) Biagioni, P.; Huang, J.-S.; Hecht, B. Rep. Prog. Phys. 2012, 75, 024402.

(7) Valev, V. K.; Baumberg, J. J.; Sibilia, C.; Verbiest, T. Adv. Mater. 2013, 25, 2517-2534.

(8) Chang, C. M.; Tseng, M. L.; Cheng, B. H.; Chu, C. H.; Ho, Y. Z.; Huang, H. W.; Lan, Y.-C.; Huang, D.-W.; Liu, A. Q.; Tsai, D. P. Adv. Mater. 2013, 25, 1118-1123.

(9) Kildishev, A. V.; Boltasseva, A.; Shalaev, V. M. Science 2013, 339, 1232009.

(10) Eggleston, M. S.; Messer, K.; Zhang, L.; Yablonovitch, E.; Wu, M. C. Proc. Natl. Acad. Sci. U. S. A. 2015, 112, 1704-1709.

(11) Yin, X.; Ye, Z.; Rho, J.; Wang, Y.; Zhang, X. Science 2013, 339, 1405-1407.

(12) Shaltout, A.; Liu, J.; Kildishev, A.; Shalaev, V. Optica 2015, 2, $860-863$.

(13) Luo, W.; Xiao, S.; He, Q.; Sun, S.; Zhou, L. Adv. Opt. Mater. 2015, 3, 1102-1108.

(14) Liu, Y.; Ling, X.; Yi, X.; Zhou, X.; Chen, S.; Ke, Y.; Luo, H.; Wen, S. Opt. Lett. 2015, 40, 756-759.

(15) Li, Y.; Liu, Y.; Ling, X.; Yi, X.; Zhou, X.; Ke, Y.; Luo, H.; Wen, S.; Fan, D. Opt. Express 2015, 23, 1767-1774.

(16) Veksler, D.; Maguid, E.; Shitrit, N.; Ozeri, D.; Kleiner, V.; Hasman, E. ACS Photonics 2015, 2, 661-667.

(17) Maguid, E.; Yulevich, I.; Veksler, D.; Kleiner, V.; Brongersma, M. L.; Hasman, E. Science 2016, 352, 1202-1206.

(18) Shitrit, N.; Yulevich, I.; Kleiner, V.; Hasman, E. Appl. Phys. Lett. 2013, 103, 211114.

(19) Petersen, J.; Volz, J.; Rauschenbeutel, A. Science 2014, 346, 6771.

(20) Drezet, A.; Genet, C.; Laluet, J.-Y.; Ebbesen, T. W. Opt. Express 2008, 16, 12559-12570.

(21) Gansel, J. K.; Thiel, M.; Rill, M. S.; Decker, M.; Bade, K.; Saile, V.; von Freymann, G.; Linden, S.; Wegener, M. Science 2009, 325, $1513-1515$.

(22) Wu, S.; Zhang, Z.; Zhang, Y.; Zhang, K.; Zhou, L.; Zhang, X.; Zhu, Y. Phys. Rev. Lett. 2013, 110, 207401.

(23) Black, L.-J.; Wang, Y.; de Groot, C. H.; Arbouet, A.; Muskens, O. L. ACS Nano 2014, 8, 6390-6399.

(24) Kuwata-Gonokami, M.; Saito, N.; Ino, Y.; Kauranen, M.; Jefimovs, K.; Vallius, T.; Turunen, J.; Svirko, Y. Phys. Rev. Lett. 2005, 95, 227401.

(25) Fedotov, V. A.; Mladyonov, P. L.; Prosvirnin, S. L.; Rogacheva, A. V.; Chen, Y.; Zheludev, N. I. Phys. Rev. Lett. 2006, 97, 167401.

(26) Schwanecke, A. S.; Fedotov, V. A.; Khardikov, V. V.; Prosvirnin, S. L.; Chen, Y.; Zheludev, N. I. Nano Lett. 2008, 8, 2940-2943.

(27) Gorodetski, Y.; Shitrit, N.; Bretner, I.; Kleiner, V.; Hasman, E. Nano Lett. 2009, 9, 3016-3019.

(28) Valev, V. K.; Smisdom, N.; Silhanek, A. V.; De Clercq, B.; Gillijns, W.; Ameloot, M.; Moshchalkov, V. V.; Verbiest, T. Nano Lett. 2009, 9, 3945.

(29) Hopkins, B.; Poddubny, A. N.; Miroshnichenko, A. E.; Kivshar, Y. S. Laser Photonics Rev. 2016, 10, 137-146.

(30) Lu, X.; Wu, J.; Zhu, Q.; Zhao, J.; Wang, Q.; Zhan, L.; Ni, W. Nanoscale 2014, 6, 14244-14253.

(31) De Leon, I.; Horton, M. J.; Schulz, S. A.; Upham, J.; Banzer, P.; Boyd, R. W. Sci. Rep. 2015, 5, 13034.

(32) Plum, E.; Liu, X. X.; Fedotov, V. A.; Chen, Y.; Tsai, D. P.; Zheludev, N. I. Phys. Rev. Lett. 2009, 102, 113902.

(33) Plum, E.; Fedotov, V. A.; Zheludev, N. I. J. Opt. A: Pure Appl. Opt. 2009, 11, 074009.

(34) Curto, A. G.; Volpe, G.; Taminiau, T. H.; Kreuzer, M. P.; Quidant, R.; van Hulst, N. F. Science 2010, 329, 930-933.

(35) Novotny, L.; van Hulst, N. Nat. Photonics 2011, 5, 83-90.

(36) Muskens, O. L.; Giannini, V.; Sanchez-Gil, J. A.; GomezRivas, J. Nano Lett. 2007, 7, 2871-2875.

(37) Kinkhabwala, A.; Yu, Z.; Fan, S.; Avlasevich, Y.; Mullen, K.; Moerner, W. E. Nat. Photonics 2009, 3, 654-657.
(38) Chung, H. Y.; Leung, P. T.; Tsai, D. P. Opt. Express 2013, 21, 26483-26492.

(39) Pirruccio, G.; Ramezani, M.; Rodriguez, S. R.-K.; Rivas, J. G. Phys. Rev. Lett. 2016, 116, 103002.

(40) Curto, A. G.; Taminiau, T. H.; Volpe, G.; Kreuzer, M. P.; Quidant, R.; van Hulst, N. F. Nat. Commun. 2013, 4, 1750.

(41) Akselrod, G. M.; Argyropoulos, C.; Hoang, T. B.; Ciracì, C.; Fang, C.; Huang, J.; Smith, D. R.; Mikkelsen, M. H. Nat. Photonics 2014, 8, 835-840.

(42) Raziman, T. V.; Martin, O. J. F. J. Phys. Chem. C 2016, 120, 21037.

(43) Huang, Y.-W.; Chen, W. T.; Wu, P. C.; Fedotov, V. A.; Zheludev, N. I.; Tsai, D. P. Sci. Rep. 2013, 3, 1237.

(44) Hancu, I. M.; Curto, A. G.; Castro-López, M.; Kuttge, M.; van Hulst, N. F. Nano Lett. 2014, 14, 166.

(45) Kruk, S. S.; Decker, M.; Staude, I.; Schlecht, S.; Greppmair, M.; Neshev, D. N.; Kivshar, Y. S. ACS Photonics 2014, 1, 1218-1223.

(46) Ren, M.; Chen, M.; Wu, W.; Zhang, L.; Liu, J.; Pi, B.; Zhang, X.; Li, Q.; Fan, S.; Xu, J. Nano Lett. 2015, 15, 2951-2957.

(47) Langguth, L.; Schokker, A. H.; Guo, K.; Koenderink, A. F. Phys. Rev. B: Condens. Matter Mater. Phys. 2015, 92, 205401.

(48) Meinzer, N.; Hendry, E.; Barnes, W. L. Phys. Rev. B: Condens. Matter Mater. Phys. 2013, 88, 041407.

(49) Cotrufo, M.; Osorio, C. I.; Koenderink, A. F. ACS Nano 2016, 10, 3389-3397.

(50) Smirnova, D.; Kivshar, Y. S. Optica 2016, 3, 1241-1255.

(51) Osorio, C. I.; Mohtashami, A.; Koenderink, A. F. Sci. Rep. 2015, 5, 9966.

(52) Kern, A. M.; Martin, O. J. F. J. Opt. Soc. Am. A 2009, 26, 732740.

(53) Mühlig, S.; Menzel, C.; Rockstuhl, C.; Lederer, F. Metamaterials 2011, 5, 64-73.

(54) Saleh, B. E. A.; Teich, M. C. Fundamentals of photonics, 2nd ed.; Wiley: Hoboken, 2007.

(55) Hellen, E. H.; Axelrod, D. J. Opt. Soc. Am. B 1987, 4, 337-350.

(56) Gorkunov, M. V.; Ezhov, A. A.; Artemov, V. V.; Rogov, O. Y.; Yudin, S. G. Appl. Phys. Lett. 2014, 104, 221102.

(57) Kataja, M.; Pourjamal, S.; van Dijken, S. Opt. Express 2016, 24, $3562-3571$.

(58) Hell, S. W.; Wichmann, J. Opt. Lett. 1994, 19, 780.

(59) Pluta, M. In Nomarski's DIC microscopy: a review; Proc. SPIE, 1994; pp 10-25.

(60) Thyagarajan, K.; Santschi, C.; Langlet, P.; Martin, O. J. F. Adv. Opt. Mater. 2016, 4, 871-876.

(61) Raziman, T. V.; Somerville, W. R. C.; Martin, O. J. F.; Le Ru, E. C. J. Opt. Soc. Am. B 2015, 32, 485-492.

(62) Paulus, M.; Martin, O. J. F. J. Opt. Soc. Am. A 2001, 18, 854861. 